\title{
RACE METIS ET HYBRIDES CHEZ LA CARPE
}

\author{
par le Docteur-Ingénieur I. POJOGA
}

Pour augmenter la production piscicole en étangs, il est de la première importance de disposer de sujets a croissance rapide et résistants aux maladies.

Dans le but d'obtenir une race de carpe sélectionnée, nous sommes arrivés, en Roumanie, à la conclusion que les races "Lausitz " et "Galicienne " introduites dans notre pays par les Jésuites au XVIIle siècle, sous le règne de Marie-Thérèse, présentaient les meilleurs avantages morphologinuement et physiologiquement Le pisciculteur $P$ FELDEOREANU et moi avons également reconnu, à la statior de pisciculture "Dumbrava-Sibiu", les qualités d'une carpe indigène, provenant de la rivière "Olt". douée d'une grande vivacité. Ce sont ces races que nous avons utilisées dans les croisements qui vont être étudiés.

\section{1 - RACE TYPE "DUMBRAVA-SIBIU "}

Nous avons d'abord procédé à la sélection phénotypique et génotypique des deux races ci-dessus désignees avant de les croiser, ne conservant

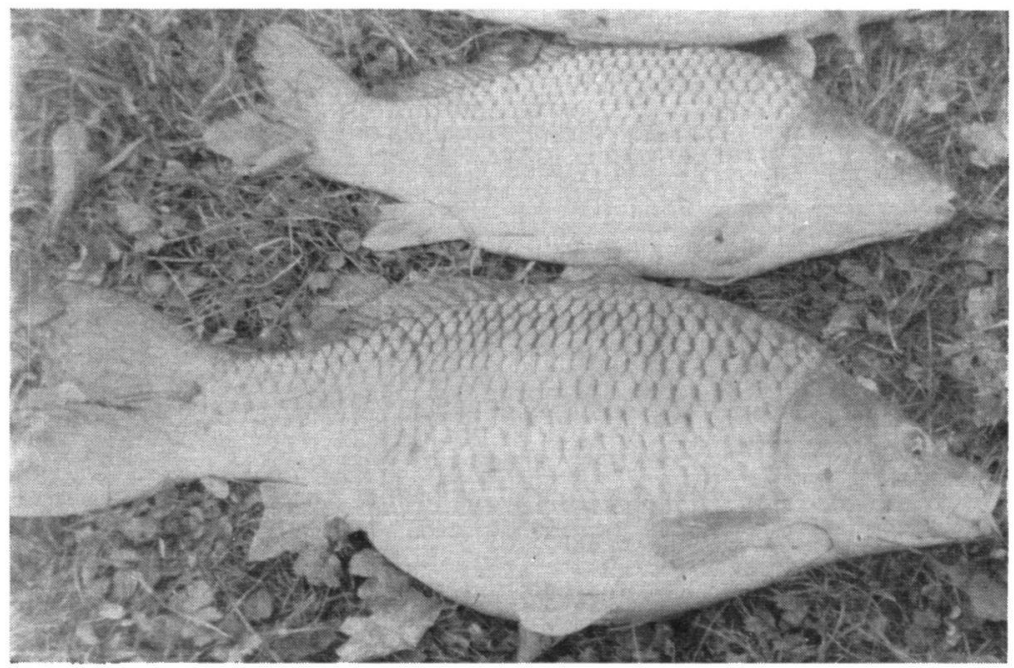

Race «Dumbrava Sibiu » 
ensuite que les métis à écailles. Cette race type, appelée "Dumbrava Sibiu *, a été obtenue au cours des étapes suivantes :

Tableau I

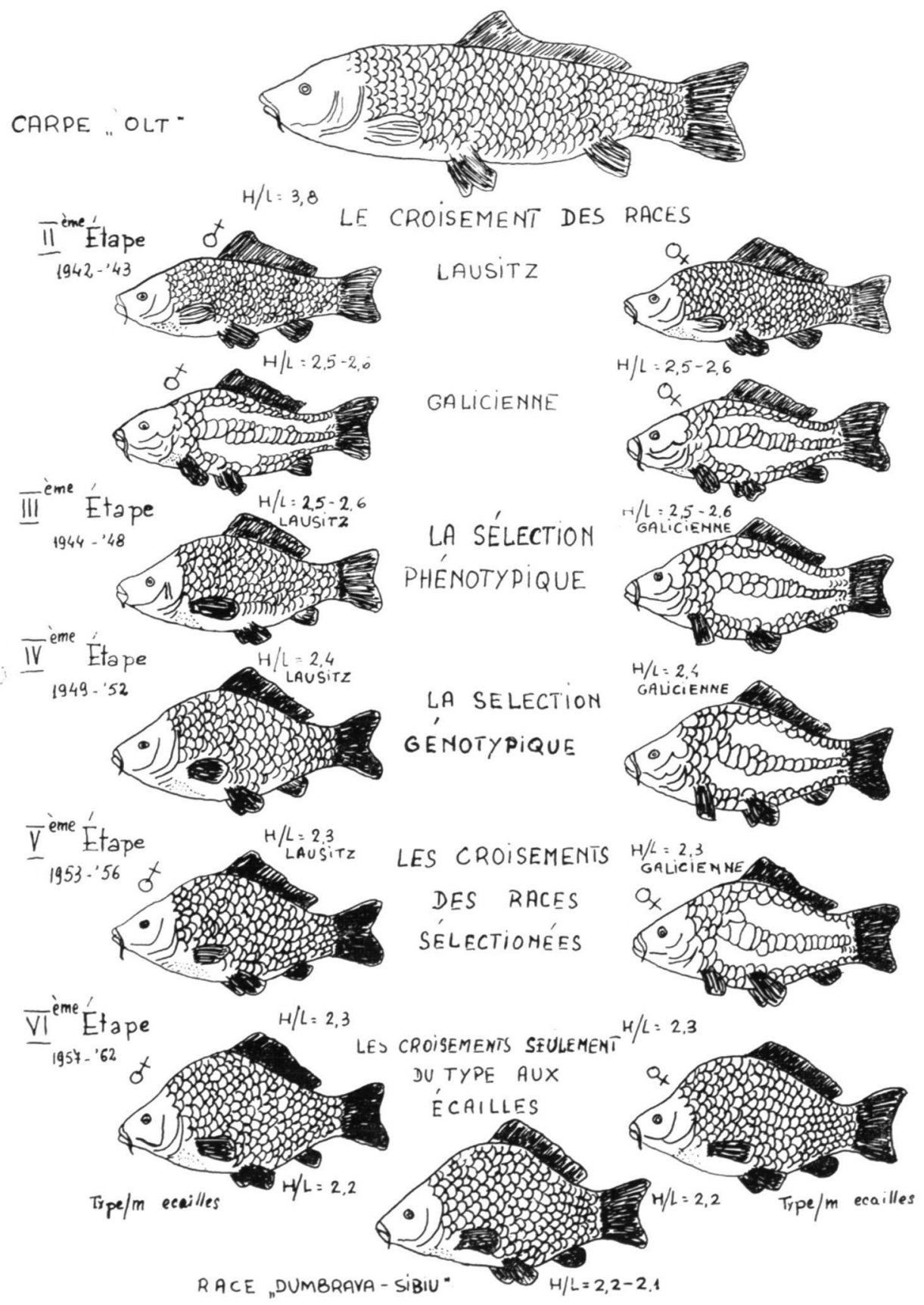

Etapes de sélection de la race "Dumbrava Sibiu» 
Tre ètape : 1940-1941:

Augmentation de la productivité des étangs dexpérience de $250 \mathrm{~kg} / \mathrm{ha}$ à $400 \mathrm{~kg} /$ ha (en moyenne), par vidange hivernale. désinfection à la chaux et fertilisation par des engrais, tant minéraux que naturels.

2e étape : 1942-1943:

Poursuite dans des étangs distincts des élevages des races Lausitz et Galicienne ayant l'indice de profil $\mathrm{l} / \mathrm{H}$ ( $\mathrm{l}$ étant la longueur du corps, à l'excep-

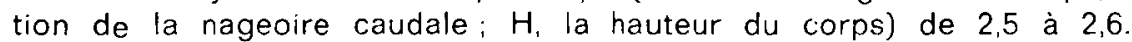

\section{3 ètape : 1944-1948:}

Selection phénotypique des sujets des deux races ayant l'indice de profil 2,4 (environ 10000 exemplaires $C_{1}$ choisis).

4 étape : 1949-1952 :

Sélection génotypique des sujets précédemment choisis. Environ $98 \%$ dentre eux avaient l'indice de profil 2,3 et plus rarement 2,4 .

$5^{e}$ étape : 1953-1956 :

Croisement des $\mathrm{C}_{3}+$ et $\mathrm{C}_{4}+$ entre mâles Lausitz et femelles Galiciennes. Les $C_{1}$ obtenus avaient l'indice de profil $2,2-2,3 ; 5 \%$ d'entre eux seulement conservant l'indice 2,4 .

6e étape : 1957-1962 :

Reproduction consanguine des seuls métis à écailles $C_{3}$. Les descendants dont très peu revêtaient la robe "miroir " avaient l'indice de profil 2,2, quelquefois même l'indice 2,1.

Voici d'ailleurs, dans le tableau ci-dessous, quelques caractéristiques de la race-type "Dumbrava Sibiu", ainsi obtenue :

\begin{tabular}{|c|c|c|c|c|c|c|c|c|c|}
\hline & Type & Sexe & Age & $\begin{array}{l}\text { Long } \\
\text { totale } \\
L \mathrm{~cm}\end{array}$ & $\begin{array}{l}\text { Long } \\
\text { sans } \\
\text { la } \\
\text { cau- } \\
\text { dale } \\
\text { L cm }\end{array}$ & $\begin{array}{l}\text { Haut. } \\
\text { du } \\
\text { corps } \\
\mathrm{H} \mathrm{cm}\end{array}$ & $\begin{array}{l}\text { Long } \\
\text { tête } \\
\mathrm{cm}\end{array}$ & $\mid \begin{array}{c}\text { Indice } \\
\text { de } \\
\text { profil } \\
/ / H\end{array}$ & $\begin{array}{l}\text { Poids } \\
\text { en kg }\end{array}$ \\
\hline Carpe & miroir & Fém. & $\mathrm{C}_{3}+$ & 54 & 43 & 19 & 11 & 2,2 & 3,340 \\
\hline Carpe & avec écaille & Mas. & $\mathrm{C}_{4}+$ & 66 & 52 & 23 & 12 & 2,2 & 4,750 \\
\hline Carpe & avec écaille & Mas. & $\mathrm{C}_{3}$ & 40 & 33 & 15 & 9 & 2,2 & 1,850 \\
\hline Carpe & avec écaille & Fèm. & $\mathrm{C}_{3}$ & 42 & 34 & 15 & 9,52 & 2,2 & 1,985 \\
\hline Carpe & avec écaille & Fém. & $\mathrm{C}_{2}$ & 28 & 23 & 11 & 6,5 & 2,1 & 0,680 \\
\hline Carpe & avec écaille & Fém. & $\mathrm{C}_{2}$ & 29 & 25 & 11 & 7 & 2,2 & 0,850 \\
\hline Carpe & avec écaille & Fém. & $\mathrm{C}_{2}$ & 33 & 27 & 12 & 7,5 & 2,2 & 0,860 \\
\hline Carpe & miroir & & $\mathrm{C}_{2}$ & 31 & 26 & 11 & 6,5 & 2,1 & 0,820 \\
\hline Carpe & miroir & & $\mathrm{C}_{2}$ & 27 & 23 & 10 & 7 & 2,1 & 0,780 \\
\hline Carpe & miroir & & $C_{1}$ & 15 & 12 & 5,5 & 4,3 & 2,2 & 0,060 \\
\hline Carpe & miroir & & $\mathrm{C}_{1}$ & 14 & 11 & 5 & 3,5 & 2,2 & 0,070 \\
\hline Carpe & avec écaille & & $\mathrm{C}_{1}$ & 16 & 14 & 6,5 & 5 & 2,2 & 0,080 \\
\hline Carpe & avec écaille & & $\mathrm{C}_{1}$ & 20 & 16 & 7 & 5 & 2,2 & 0,170 \\
\hline Carpe & avec écaille & & $C_{1}$ & 19 & 15 & 7 & 4,5 & 2,2 & 0,180 \\
\hline Carpe & avec écaille & & $\mathrm{C}_{1}$ & 21 & 18 & 8 & 4,5 & 2,2 & 0,180 \\
\hline Carpe & avec écaille & & $\mathrm{C}_{1}$ & 15,5 & 12,5 & 5,5 & 3,8 & 2,2 & 0,070 \\
\hline Carpe & avec écaille & & $C_{1}$ & 23,5 & 19,5 & 9,5 & 5 & 2 & 0,280 \\
\hline Carpe & avec écaille & & $\mathrm{C}_{1}$ & 24 & 20 & 10 & 6,5 & 2 & 0,310 \\
\hline
\end{tabular}


Les sujets $C_{11}$ et $C_{1}$ ont été nourris par apport d'aliments supplémentaires; on a reconnu que la nourriture naturelle convenait mieux aux sujets destinés à la reproduction.

La valeur économique de la race ainsi obtenue a été prouvée par la comparaison des rendements suivants notés dans les étangs d'expérience 5 , 6 et 7 .

Etang no 5 - 2,2 ha : Métis Lausitz $x$ Carpe sauvage Olt. Production de $\mathrm{C}_{1}+$ de $2282 \mathrm{~kg}$, soit $1070 \mathrm{~kg}$ à l'ha.

Etang no $6-1,7$ ha : Carpes Lausitz et Galiciennes adaptees aux conditions locales. Production de $C_{1}+$ de $1650 \mathrm{~kg}$, d'un poids moyen par unité de $490 \mathrm{~g}$, soit $987 \mathrm{~kg} / \mathrm{ha}$. Sibiu.

Etang no 7 - 1 ha : Production de $1468 \mathrm{~kg} /$ ha de carpe métis Dumbrava

Le rendement ètait donc augmenté de $3,5 \%$ par rapport à celui obtenu chez les métis Lausitz $x$. Olt et de $37 \%$ par comparaison aux étangs à Lausitz $\times$. Galicienne.

\section{2 - METIS CARPE OLT $\times$ CARPE LAUSITZ. OU GALICIENNE}

C'est un fait bien connu que les produits de croisement ont généralement une vigueur p!us grande que celle des parents. $C e$ phénomène est appelé hétérosis. On l'attribue soit a la compensation des facteurs semi-léthaux qui peuvent exister chez les parents, soit à la réunion de facteurs favorables dont les effets s'ajoutent.

De nombreux naturalistes, DARWIN, WALTON, HAMNOD, CHAT, MORLEY, SM!TH, SACHY, DUBINI, se sont occupés de ce phénomène, sans parvenir à une théorie acceptable par tous. DINU et TURCU estiment que l'apparition de l'hétérosis dópend uniquement des races utilisées pour l'accouplement.

Quoiqu'ii en soit, nous avons procédè, à Dumbrava Sibiu, en collaboration avec le pisciculteur P FELDEOREANU, à des croisements entre la Carpe sauvage de la rivière Olt et la Carpe de race Lausitz bien adaptée aux conditions locales.

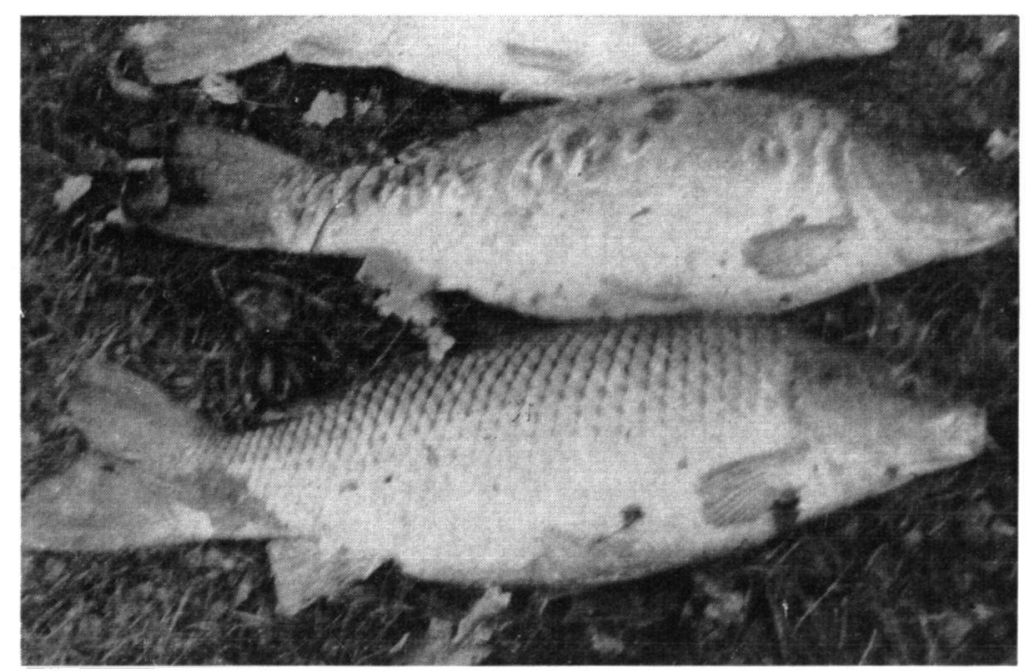

Métis "Dumbrava Sibiu" 
Les caractéristiques de cette dernière étaient les suivantes :

- Indice de profil : 2,4 à 2,6.

- Formule des nageoires : A III 6, DIII 19 (21), VII9, PI 16.

- Ecailles sur la ligne laterale : (34) $355 / 631$.

- Dents pharyngiennes : $1.1 .3-3.1 .1$.

- Branchiospines : 29 (30).

- Vertèbres : 36 (37).

- Côtes : 15 (16).

- Lonjueur du corps (sans la nageoire caudale) : 3,8 à 1,5 fois plus grande que celle de la tête.

- Poids des oviles au IVe stade (fin avril) : 17,6 à $19,9 \%$ du poids total du corps.

Les caractéristiaues de la Carpe de Olt qui appartient à la variété - hungaricus", au corps allongé, presque cylindrique, étaient les suivantes : - Indice de prof:l : 3,3 à 3,5 .

- Formule des nageoires : A III 5 (6), DIII 20 (21), VII 8, PI 17.

- Ecailles sur la ligne latérale : $37 \quad 5 / 6 \quad 38$ (39).

- Branchiospines : 25 (26).

- Dents pharyngiennes : $1.1 .3-3.1 .1$.

- Vertèbres : 36 (37).

- Côtes : 16 (15).

- Longueur du corps (sans la nageoire caudale) : 4 à 4,5 fois plus grande que celle de la tête.

- Poids des ovules au IVe stade (fin avril) : 19 à $23 \%$ du poids total du corps.

La Carpe "O!t " est caractérisée par une grande vitalité; elle a une aptitude marquée pour rechercher la nourriture naturelle.

A l'échelle semi-industrielle, les croisements entre femelles Lausitz et mâles Olt ont commencé en 1948. Les métis obtenus dont la majorité portait des écailles $(95 \%)$, les autres étant du type "miroir *, avaient les caractéristiques suivantes (pour certains d'entre eux) :

\begin{tabular}{|c|c|c|c|c|c|c|}
\hline Age & $\begin{array}{c}\text { Longueur } \\
\text { totale } \\
\text { L cm }\end{array}$ & $\begin{array}{c}\text { Long. } \\
\text { sans la } \\
\text { caudale } \\
\mathrm{Icm}\end{array}$ & $\begin{array}{c}\text { Hauteur } \\
\text { du corps } \\
\mathrm{H} \mathrm{cm}\end{array}$ & $\begin{array}{c}\text { Long } \\
\text { de la tête } \\
\mathrm{cm}\end{array}$ & $\begin{array}{c}\text { Indice de } \\
\text { profil } \\
\mathrm{I} / \mathrm{H}\end{array}$ & $\begin{array}{c}\text { Poids } \\
\text { en kg }\end{array}$ \\
\hline M 5 & 64,0 & 51 & 18 & 13,0 & 2,7 & 4,000 \\
M 3 & 41,0 & 35 & 15 & 12,3 & 2,7 & 1,000 \\
M2 & 34,0 & 28 & 10 & 8,0 & 2,7 & 0,840 \\
M2 & 27,5 & 24 & 11 & 8,0 & 2,7 & 0,890 \\
M2 & 31,0 & 27 & 10 & 7,0 & 2,7 & 0,540 \\
\hline
\end{tabular}

- Formule des nageoires : A III 5 (6), DIII 21 (20), VII 7, PI 17.

- Ecailles de la ligne latérale : $375 / 638$ (39).

- Branchiospines : 29 (30).

- Vertèbres : 35 (36).

- Côtes : 16 (15).

- Longueur du corps sans la nageoire caudale : 3,9 à 4 fois celle de la tête. 


\section{Tableau II}

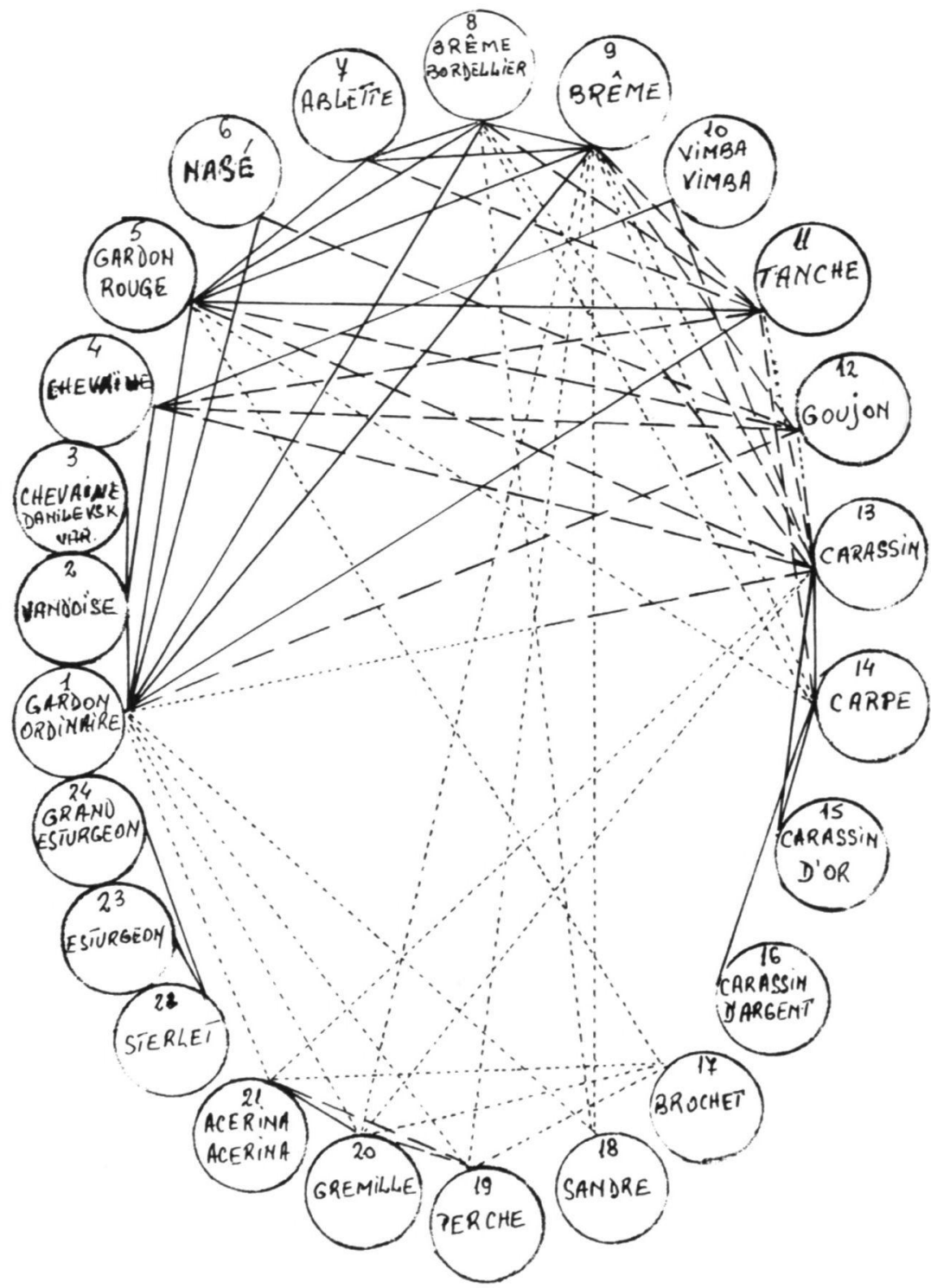

Les hybrides chez les poissons

\section{Légende :}

(traits pleins) : hybrides existants ;

- - (traits entrecoupés) : hybrides obtenus, mais non conservés; (pointillés) : hybrides non obtenus. 
Les métis avaient donc, à la fois des caractères "Lausitz * et des caractères "Olt"; le corps était plus allongé que celui de la "Lausitz *, mais l'indice de profil se montrait plus voisin de cette dernière; le nombre des écailles de la ligne latérale, la couleur, la forme des écailles et des nageoires. le squelette étaient proches de la race "Olt $n$, sauf la tête plus petite.

Au point de vue physiologique, ces métis se caractérisaient par une grande vivacité, héritée de cette dernière race.

Transportés dans les étangs de la Coopérative Agricole de Production (C.A.P.) de la région de Bucarest (Lupsanu, Nucet, Radu-Voda, et Dor Marent, arrondissement de Lehliu), ils se sont montrés résistants à l'hydropisie infectieuse, alors que cette épidémie avait èté signalée fréquemment auparavant. La reproduction, comparèe à celle notée pour la Carpe locale, avait èté augmentée de $22 \%$.

\section{3 - HYBRIDES ENTRE LA CARPE ET LE CARASSIN DORE}

A côté des métis produits par des individus d'une même espèce. mais de race différente, il existe des hybrides issus du croisement d'espèces différentes ou même de genres différents.

Ces hybrides, d'abord souvent décrits comme des espèces à part (SIEBOLD 1863, HEKEL 1864, 1866, 1870, etc.), ont eu leur véritable nature découverte dans la deuxième moitiè du XIXe siècle (KESSLER 1879, BONKE 1884, KNAUTHE 1893, 1896, etc.).

C'est ainsi que, chez le genre Acipenser (esturgeon), les hybrides sont nombreux. Décrits pour la première fois en 1909 par ANTIPA GREGORE, ils ont été obtenus, en utilisant la fécondation artificielle, par KOWALEVSKI, OWSJANIKOV et WAGNER, et NICOLIUKIN a montré (1949-1960) que, rarement féconds, ils se croisaient dans les conditions naturelles avec les formes parentales, presoue jamais entre eux.

$1 /$ existe également un hybride de genre entre la Carpe (Cyprinus carpio) et le Carassin doré (Carassius auratus). Nous l'avons décrit, avec E. COSTEA pour la première fois, en Roumanie, dans les conditions naturelles en 1955. Dans le but de posséder des sujets à croissance rapide, à régime nutritif différent de celui de la Carpe, à chair plus savoureuse que celle des parents, tout en ètant plus résistants aux maladies, nous avons procédé à une telle hybridation avec TH. BUSNITA et E. COSTEA.

La Carpe utilisée a été une femelle sauvage, provenant de * Balta greaca $n$, âgée de 7 ans, longue de $75 \mathrm{~cm}$ et pesant $5300 \mathrm{~g}$; les deux mâles de Carassin doré étaient issus du jardin "Cismijiu" de Bucarest; âgés de 3 ans, ils mesuraient $16 \mathrm{~cm}$ et pesaient $55 \mathrm{3}$. On procéda par fécondation artificielle avec injection hypophysaire et l'on obtint, quinze jours après l'éclosion, 45000 alevins environ, d'une longueur de 1,5 à $2 \mathrm{~cm}$, dont un millier furent placés à la station * Nacet " dans un étang de 2 ha, auprès d'alevins de Carpe d'èlevage. On pècha, l'automne suivant. 400 hybrides d'une longueur moyenne de $21 \mathrm{~cm}$ et d'un poids moyen de $210 \mathrm{~g}$, alors que les jeunes Carpes d'élevage, du même âge, mesuraient $22 \mathrm{~cm}$ et ne pesaient en moyenne que $180 \mathrm{~g}$. 
Les caractéristiques de la Carpe de lac "greaca "étaient celles de la Carpe du Danube :

- Indice de profil : 3,5.

- Formule des nageoires : D III 21. A III 6, V II 9, PI 16.

- Ecailles de la ligne latérale : 37 5/5 38 .

- Branchiospines : 23.

- Dents pharyngiennes: $1.1 .3-3.1 .1$.

- Vertèbres : 35.

- Côtes : 15

- Longueur de l'intestin : 1.9 fois la longueur du corps. sans la nageoire caudale.

- Deux paires de gros barbillons.

- Couleur des écailles : or foncé.

Le Carassin doré était ainsi caractérise :

- Indice de profil : 2,4.

- Formule des nageoires : D III 17, All6, Vi 7, PI 15.

- Ecailles de la ligne latérale : 30 6/7 30.

- Branchiospines : 38.

- Dents pharyngiennes : 4 - 4 .

- Vertèbres : 28.

- Côtes : 15

- Longueur de l'intestin : 2,6 fois la longueur du corps, sans la nageoire caudale.

- Couleur des écailles : orange rougeâtre.

L'hybride obtenu possedait les caracteres intermédiaires suivants

- Indice de profil : 2,4 - 2,5.

- Formule des nageoires : D III 21, A II6(5), VI7, PI 15.

- Ecailles de la ligne latéraie : 35 (34 - 6/7. 24) (35).

- Dent's pharyngiennes : $1-4-4-1-4-4$.

- Branchiospines : 34 (33).

- Vertèbres : 34 (35).

- Côtes : 15

- Longueur de lintestin : 2,6 fois la longueur du corps, sans la nageoire caudale.

Tête ressemblant à celle de la carpe mais hauteur du corps plus grande. Bouche à lères minces comme chez le Carassin doré; deux paires de très petits barbillons dont la premiére paire réduite parfois à de petits boutons, quelquefois une seule paire de barbillons. Nombre des écailles de la ligne latérale intermédiaire entre celui de la Carpe et celui du Carassin. Nageoires semblables à celles de la Carpe. Couleur argentée aux reflets roses. Nombre des rayons de la nageoire dorsale semblable à celui de la Carpe, come le nombre des vertèbres. Intestin long et mince du Carassin.

Le régime alimentaire de l'hybride se rapprochait de celui de cette dernière espèce, composé de préférence de végétaux, surtout très petits, retenus sur le filtre formé par les nombreuses branchiospines. 
La resistance aux transports comme au manque d'oxygène était plus grande que celle de ses parents. Malheureusement il ne supportait pas l'hydropisie des Cyprinides (Septicemia hemoragiium Cyprinorum), contractant toujours la forme aigue caractéristique du Carassin doré. Au printemps 1953, les hybrides d'un an etaient atteints dans la proportion de $20 \%$.

En 1955 on répéta ces hybridations à la station de Nucet, en utilisant cette fois la Carpe l.ausitz. Les sujets qu'on obtint, élevés avec des individus de race, avaient atteint apres deux etés une longueur de $30 \mathrm{~cm}$ et un poids moyen de 550 grammes avec un développement aussi bon que celui de la carpe de race, mais $80 \%$ d'entre eux furent atteints d'hydropisie en mai 1956 et $30 \%$ en moururent.

En 1956, on procéda au croisement inverse : des Carpes mâles et des Carassins femelles, mais les hybrides qui en résulterent ne se montrèrent pas plus résistants à l'hydropisie.

E. COSTEA et A. CRISTIAN (1962) ont constaté qu'introduit en étang comme poisson de supplément l'hybride Carpe $\times$ Carassin etait susceptible d'augmenter la production de $23 \%$, par comparaison à celle notée en utilisant le Carassin argenté (Carassius auratus gibelio - BLOCH). Ils ont recommandé de l'introduire en automne dans des ètangs contenant des fleurs d'eau, pour diminuer ces dernières et éviter la contagion de l'hydropisie.

\section{4 - CONCLUSION}

Des recherches effectuées en Roumanie sur les hybrides de genres Carpe $\times$ Carassin doré et les hybrides d'espèces Carassin doré $\times$ Carassin, on peut tirer les conclusions suivantes.

Les hybrides Carpe $\times$ Carassin doré se montrent :

- en général, inféconds (1);

- plus résistants que la Carpe au régime déficitaire en oxygène, tout en ayant un développement semblable à celui de la Carpe ;

- plus réceptifs que cette espèce à l'hydropisie infectieuse;

- sans intérèt pour un élevage industriel, leur production étant difficile, onéreuse et pratiquement sans rentabilité.

Quant aux hybrides entre les espèces du genre Carassius (Carassius auratus $\times$ Carassius carassius), étudiés par TH. BUSNITA, E. COSTEA et I. POJOGA en 1967. ils ont manifesté un pourcentage de fécondation des œufs plus élevé que les hybrides de genres Carpe $\times$ Carassin doré. Leur croissance a èté meilleure que celle de leurs parents.

Les métis de Carpe ont manifesté, d'après nos observations personnelles, à l'hydropisie infectieuse

- une résistance totale, pour la race « Dumbrava Sibiu», étudiée plus haut;

- une résistance relative pour les métis "Lausitz $\times$ Siut-Ghiol; ceux-ci ont été atteints. dans les conditions normales, dans la proportion de 0.5 à $1 \%$, au lieu de quelquefois $8 \%$ pour la Carpe de race.

(1) Cependant KISILIEV a réussi, en 1958, à obtenir des descendants d'une femelle iéconde. Quelques-uns de ces hybrides sont hermaphrodites. 


\section{ANALYSE BIBLIOGRAPHIQUE}

DECAMPS H., 1971 - La vie dans les cours d'eau - Collection * Que sais-je ? * Presses universitaires de France, 108, Boulevard Saint-Germain, Paris, 128 pp.

L'ouvrage que vient de publier M. Henri DECAMPS, chargè de recherches au C.N.R.S., est tout à fait d'actualité : quel rôle ont les êtres vivants dans les cours d'eau? * Quelle fonction ont-ils dans la maintenance de l'équilibre de la vie? Comment contribuent-ils à l'évolution des communautés? Quelle place occupent-ils dans la chaine alimentaire qui, par le poisson, conduit finalement à l'homme? La réussite des études de pollution et d'aménagement dépend de ces questions."

Nous n'avions pu dans notre livre : * La vie dans les eaux douces * (1) consacré surtout aux lacs. qu'effeurer les problèmes spéciaux posés par les cours d'eau. Ces dernières années, de nombreuses recherches ont été faites sur ceux-ci, tant en France qu'à l'étranger et en particulier par l'auteur lui-même : l'essentiel nous en est donné dans ce livre, clair, précis et d'une lecture aisée.

La jre partie est consacrée aux conditions particulières de la vie en eau courante, bien différentes de celles des lacs. Lo débit les caractérise, variable avec le climat, la perméabilité du terrain, la pente; la composition chimique est le résultat de celle des terrains traversés. La couverture végétale a une action marquée : elle agit soit directement par l'ombre que les végétaux projettent ou par les feuilles accumulées en automne, soit indirectement par l'action du déboisement sur l'écoulement des eaux. Dans une même section transversale du cours d'eau. le courant lui-même présente des vitesses différentes, les plus rapides se situent près de la surface, les plus faibles près des rives ou du fond : il agit de façon variée sur le comportement des animaux qui doivent lutter plus ou moins contre lui; en modifiant par le transport ou le dépôt les éléments du substrat, if conditionne aussi la structure du fond et par voie de conséquence celle des communautés qui l'habitent; enfin il assure l'évacuation rapide des déchets en facilitant les échanges gazeux, le renouvellement des substances dissoutes et l'apport des produits alimentaires. Par suite d'un enrichissement progressif en matières nutritives vers l'aval, la production d'un cours d'eau et principalement la production primaire va en augmentant dans cette direction.

Bien entendu, un cours d'eau présente des aspects ou biotopes variés suivant la nature du fond : pierreux, caillouteux, sableux, argileux, limoneux ou vaseux et suivant les végétaux qui s'y trouvent. En profil longitudinal, on distingue trois grandes régions: la partie voisine de la source ou crénon, la partie supérieure ou rhitron, la partie inférieure ou potamon, caractérisées par des différences d'amplitudes thermiques.

La $2^{e}$ partie de l'ouvrage traite des communautés d'eau courante, qu'on peut répartir, de l'amont vers l'aval, en plusieurs zones; celles-ci sont bien connues pour les poissons (zone à truite, à ombre, à barbeau, à brême), plus discutables pour les invertébrés (classification d'ILIES et BOTOSANEANU).

(1) Collection * Que sais-je? * - 3e Edition - 1961 
Les animaux sont constamment en dénlacements, soit sous l'action de tactismes (phototactisme, rhéotactisme, stéréotactisme), soit sous l'action de la dérive, soit encore sous celle de la remontée du courant ou de l'imergence des insectes adultes, soit enfin, pour certains, en raison de l'enfoncement en profondeur. Chez les poissons, en particulier, les mouvements sont en rapport avec l'alimentation et la reproduction. Chez tous les êtres, les variations saisonnières sont ressenties différemment suivant le temps qu'ils mettent à leur développement. ce temps pouvant ètre supérieur (poissons, écrevisses), ègal (macrophytes) ou inférieur (bactéries. microphytes) au cycle saisonnier annuel.

Les écosystèmes sont l'objet de transformations qui sont examinées dans une $3 e$ partie. Les aproducteurs " ou "décomposeurs " de la matière orqanique, végétaux, détritus, microorganismes, contribuent avec les apports exogènes au développement des "consommateurs", invertébrés ou poissons. II y a inévitablement un rapport entre les besoins alimentaires de ces derniers et la nourriture qu'ils trouvent sur le fond; les truites, à ce propos, se montrent particulièrement exigeantes puisqu'elles paraissent utiliser une nourriture six à sept fois supérieure à la biomasse moyenne d'invertébrés du fond; ce paradoxe peut s'expliquer ainsi (d'apres MANN) : "Les populations piscicoles des cours d'eau s'alimentent au taux maximal permis par le benthos présent. Ce dernier, maintenu à une faible densité: tend à augmenter sa biomasse II se renouvelle à un taux tel que sa production annuelle peut atteindre ou dépasser dix fois la biomasse moyenne?.

La production primaire des cours d'eau est plus difficile à déterminer que celle des lacs, car elle n'est pas due seulement aux organismes planctoniques, mais à des organismes plus ou moins irrégulièrement fixés sur le fond, elle n'est pas très élevée par rapport à d'autres milieux : de 0,1 à $0,6 \mathrm{~kg}$ de matière vivante par mètre carré et par an. En ce qui concerne la production piscicole proprement dite, celle en particulier des truites en eau courante, à partir d'un certain niveau de population, une augmentation de la densité ne l'augmente pas. Il existe une production maximale en rapport avec la "capacité biogénique" du cours d'eau, qu'on peut accroitre par un aménagement rationnel (apport de sels nutritifs).

Un système peut être plus ou moins bioactif, c'est-à dire plus ou moins capable, en unité de temps et de surface, de former de l'énergie potentielle et de la convertir en énergie cinétique. Nous aurons ainsi, suivant les cas, des eaux oligodynamiques dans lesquelles la matière organique, entrainée vers l'aval, n'est que partiellement déccmposée, des eaux eudynamiques. dans le cas contraire.

* Le bassin de drainage en entier influence... l'équilibre des communautés d'eau courante... La décharge d'égouts, l'érosion des sols, le lessivage des engrais accélèrent brutalement l'entrophisation des rivières $\%$ Les phénomènes sont analogues à ceux des lacs et aboutissent finalement à la disparition des espèces nobles, truites et saumons. "Toute forme de vie peut finalement disparaitre de la rivière .

Nous ne saurions trop recommander la lecture de ce livre si instructif à tous ceux qui s'intéressent à la vie aquatique et, en tout premier lieu, aux biologistes et aux pêcheurs

\section{P. VIVIER}

